\title{
Thermal Effect of Thin Elastic Plates
}

\author{
WX Zhang ${ }^{1,2 *}$ \\ ${ }^{1}$ Nantong Polytechic College, Nantong, Jiangsu, 226002, China \\ ${ }^{2}$ School of Civil and Environmental Engineering, Hunan University of Science and Engineering, Yongzhou, Hunan, 425199, China
}

\begin{abstract}
Thermal effect refers to the heat released or absorbed by the object in the changing process at a certain temperature. In this process, the stress inside the material will change. Thermal stress refers to the stress produced when the temperature changes, because of the external constraints and the internal constraints between the various parts, so that it can not completely free expansion and contraction.
\end{abstract}

\section{Introduction}

To solve the thermal stress, it is necessary to determine not only the temperature field, but also the displacement, strain and stress fields. Time independent temperature field is called steady temperature field, which causes steady thermal stress. The temperature field changing with time is called unsteady temperature field [1-3]. In the traditional method, the temperature distribution is obtained from the heat conduction equation and boundary conditions, and then the displacement and stress are calculated according to the thermoelastic equation [4].

Elastomer generally refers to the material that can be restored to its original state after removing external force. However, the material with elasticity is not necessarily an elastomer. Elasticity is an important branch of solid mechanics. It studies the deformation and internal force of elastic body under the action of external force and other external factors, also known as elastic theory. Elasticity is the basis of material mechanics, structural mechanics, plastic mechanics and some interdisciplinary subjects, and elastic theory is widely used in construction, machinery, chemical engineering, aerospace and other engineering fields. When the external force does not exceed a certain limit, the object will return to its original state after removing the external force $[5,6]$.

$$
\begin{aligned}
& \varepsilon_{x}=\frac{\partial u}{\partial x}, \varepsilon_{\theta}=\frac{1}{r} \frac{\partial v}{\partial \theta}-\frac{w}{r}, \varepsilon_{x \theta}=\frac{1}{r} \frac{\partial u}{\partial \theta}+\frac{\partial v}{\partial x}, \\
& \kappa_{x}=-\frac{\partial^{2} w}{\partial x^{2}}, \kappa_{\theta}=-\frac{1}{r^{2}} \frac{\partial^{2} w}{\partial \theta^{2}}, \kappa_{x \theta}=-\frac{1}{r} \frac{\partial^{2} w}{\partial x \partial \theta} .
\end{aligned}
$$

The above equations should satisfy the following boundary conditions: 


$$
\begin{aligned}
& \left.w \cdot\left[D \partial_{x}^{3} w+D \frac{(1-v)}{r^{2}} \partial_{x} \partial_{\theta}^{2} w+N_{x} \partial_{x} w\right]\right|_{x=x_{0}}=-\left.w \cdot Q_{x}\right|_{x=x_{0}}=0 \\
& \left.\partial_{x} w \cdot D\left(\partial_{x}^{2} w+\frac{v}{r^{2}} \partial_{\theta}^{2} w\right)\right|_{x=x_{0}}=-\left.\phi_{x} \cdot M_{x}\right|_{x=x_{0}}=0
\end{aligned}
$$

Applying the variational principle, we have displacements and stress components

$$
\begin{gathered}
\zeta(x)=C_{1} \cos \alpha_{1 n} x+C_{3} \cos \beta_{1 n} x \\
\xi(x)=C_{1} e^{\alpha_{2 n} x} \cos \beta_{2 n} x+C_{2} e^{\alpha_{2 n} x} \sin \beta_{2 n} x \\
\eta(x)=C_{3} e^{-\alpha_{2 n} x} \cos \beta_{2 n} x+C_{4} e^{-\alpha_{2 n} x} \sin \beta_{2 n} x \\
\alpha_{1 n}=\left\{\frac{N_{x}}{D}-\frac{n^{2}}{r^{2}}+\left[\left(\frac{N_{x}}{D}-\frac{n^{2}}{r^{2}}\right)^{2}-4\left(\frac{E h}{r^{2} D}+\frac{n^{4}}{r^{4}}\right)\right]^{1 / 2}\right\}^{1 / 2} / \sqrt{2} \\
\beta_{1 n}=\left\{\frac{N_{x}}{D}-\frac{n^{2}}{r^{2}}-\left[\left(\frac{N_{x}}{D}-\frac{n^{2}}{r^{2}}\right)^{2}-4\left(\frac{E h}{r^{2} D}+\frac{n^{4}}{r^{4}}\right)\right]^{1 / 2}\right\}^{1 / 2} / \sqrt{2}
\end{gathered}
$$

So the final dual governing equation are obtained as

$$
\dot{\psi}=\left[\begin{array}{cccc}
0 & -1 & 0 & 0 \\
\partial_{x}^{2} & 0 & 0 & 1 / D \\
\frac{E h}{r^{2}}+N_{x} \partial_{x}^{2} & 0 & 0 & -\partial_{x}^{2} \\
0 & 0 & 1 & 0
\end{array}\right] \psi
$$

The symplectic orthogonal relation between the eigensolutions is

$$
\begin{aligned}
& <\varphi_{i}, \varphi_{j}>=\int_{0}^{x_{e}} \varphi_{i}^{T} J \varphi_{j} d x=0 \\
& \psi(x, \theta)=\sum_{n=0}^{\infty}\left[a_{n} \varphi_{n}(x) e^{i n \theta}+b_{n} \varphi_{-n}(x) e^{-i n \theta}\right]
\end{aligned}
$$

\section{Numerical example}

Fig. 1 and Fig. 2 exhibit stress response of the domain in a particular temperature field. The results show that the material has a strong dependence on the change of temperature. Even in the constant temperature field, the change of temperature conditions also has a significant impact on the stress and deformation of the material. 


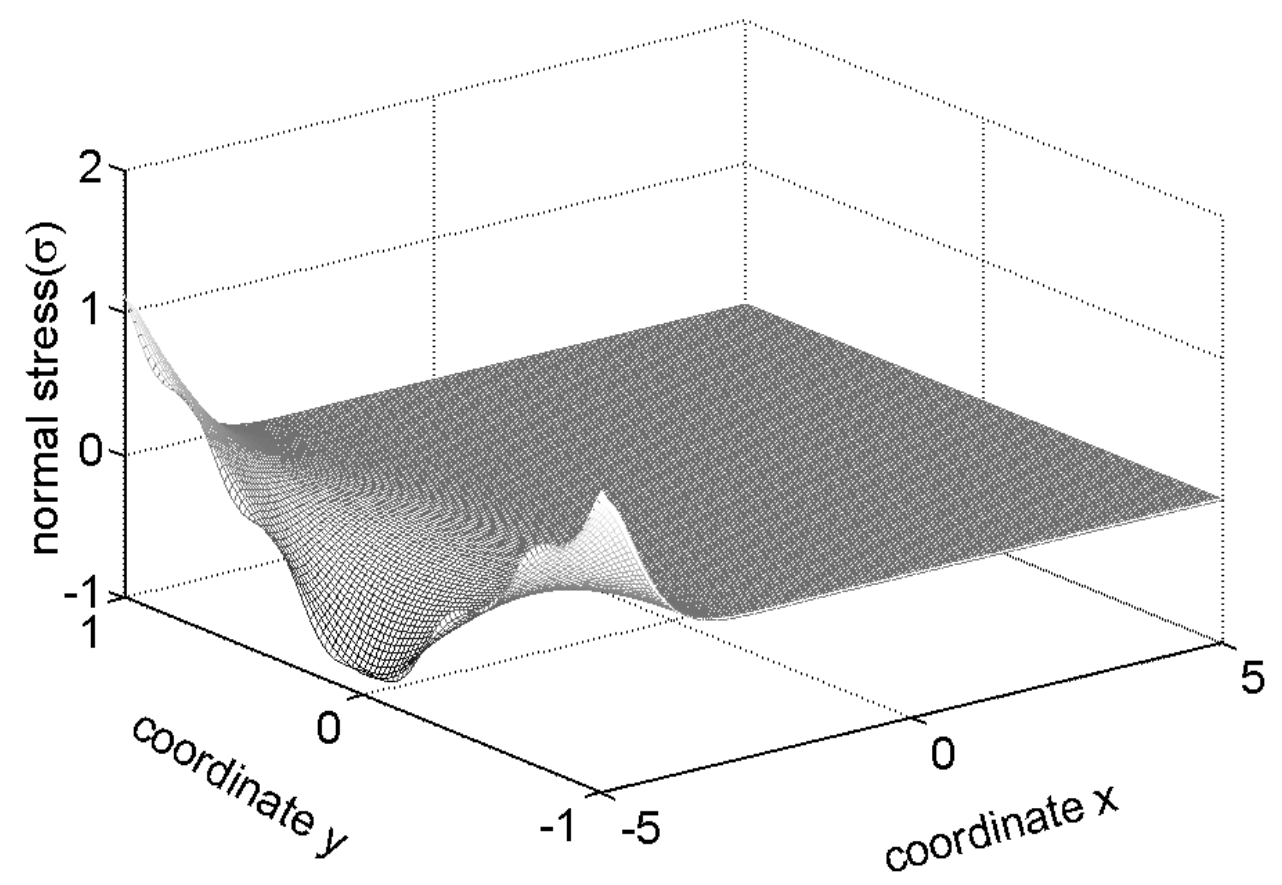

Fig 1. Normal stress

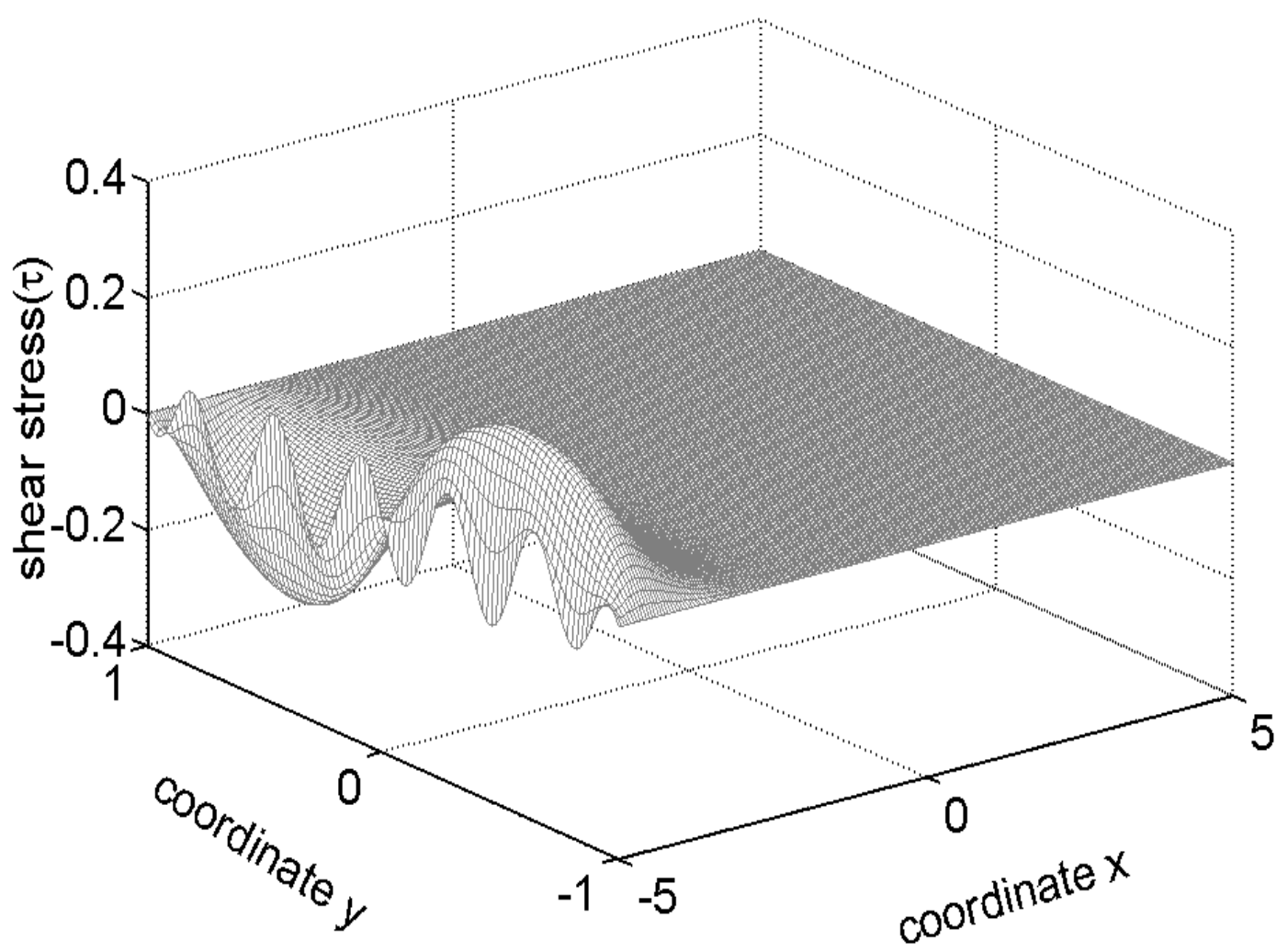

Fig 2. Shear stress

\section{Conclusion}

The elastic material is obviously dependent on the temperature condition. Based on the variational principle and Laplace integral transformation, the basic equations of thermoelasticity are transformed into solving the problem of nonhomogeneous boundary conditions. Using this technique, the stress and deformation of elastic materials in a specific temperature field are discussed. 


\section{Reference}

1. Hine, P. J., Gusev, A. A. (2019) Validating a micromechanical modelling scheme for predicting the five independent viscoelastic constants of unidirectional carbon fibre composites. Int. J. Eng. Sci., 144: 103-133.

2. Sobhy, M. (2015) Levy-type solution for bending of single-layered graphene sheets in thermal environment using the two-variable plate theory. Int. J. Mech. Sci., 90: 171-178.

3. Mircea, B. (2007). On Saint-Venant's principle in the theory of Cosserat elastic shells. Int. J. Eng. Sci., 45: 187-198.

4. Colak, O. (2005) Modeling deformation behavior of polymers with viscoplasticity theory based on overstress. Int. J. Plasticity, 21: 145-160.

5. Khan, A.S., Zhang, H. (2001) Finite deformation of a polymer and constitutive modeling. Int. J. Plasticity, 17:1167-1188.

6. Kuna, M. (1998) Finite element analyses of crack problems in piezoelectric structures. Comput. Mater. Sci., 13: 67-80. 